Cell Research (1999), 9, 163-170

\title{
MINIREVIEW \\ Regulation of eukaryotic DNA replication and nuclear structure
}

\author{
WU JIA RUI \\ Shanghai Institute of Biochemistry, Chinese Academy of \\ Sciences, Shanghai 200031, China \\ e-mail:wujr@sunm.shcnc.ac.cn
}

\begin{abstract}
In eukaryote, nuclear structure is a key component for the functions of eukaryotic cells. More and more evidences show that the nuclear structure plays important role in regulating DNA replication. The nuclear structure provides a physical barrier for the replication licensing, participates in the decision where DNA replication initiates, and organizes replication proteins as replication factory for DNA replication. Through these works, new concepts on the regulation of DNA replication have emerged, which will be discussed in this minireview.
\end{abstract}

Key words: DNA replication, nuclear structure, replication licensing, replication origin, replication factory

Regulatory mechanisms for DNA replication are central to the control of the cell-cycle in eukaryotic cells. Recently, considerable progress has been made in our understanding of the relationship between regulation of eukaryotic DNA replication and nuclear structure. This review will briefly outline the progress and discuss some new concepts appearing from the studies.

\section{Licensing of DNA replication: the initiation-competent state}

Replication of the eukaryotic genome occurs only once during the S-phase of each cell cycle. More than 20 years ago, Rao and Johnson found that DNA synthesis in G1 nuclei was induced by fusing G1-phase human cells with S-phase human cells, but no DNA synthesis in G2 nuclei was detected by fusing G2-phase cells with S-phase cells. 
Regulation of eukaryotic DNA replication and nuclear structure

Thus, G1-phase cells but not G2-phase cells are in a replication-competent state[1]. Lately, Laskey and Blow revealed that if the G2-phase nuclear membrane was permeabilized artificially, nuclei became competent for a second round of DNA synthesis[2]. They proposed a model that an essential replication factor (replication licensing factor), which allows the initiation of DNA replication to occur, can only access chromatin during mitosis after nuclear membrane breakdown. Then the factor would be inactivated during DNA replication to ensure only one round of DNA synthesis per cell-cycle[1],[2].

With a genetic screening assay in yeast, a family of physically and genetically interacting proteins have been suggested to be the putative replication licensing factor, including the MCM2, MCM3 and CDC46/MCM5 proteins from Sccharomyces cerevisiae, and NDA1, NDA4 and CDC21 from Schizosaccaromyces pombe[3],[4]. MCM proteins are essential for minichromosome maintenance in S. cerevisiae, and appear in the nucleus at the end of mitosis, remain during G1 and then disappear from the nucleus at the beginning of $\mathrm{S}$ phase. By using biochemical assays, three laboratories subsequently reported the identification of components of the licensing factors in higher eukaryotic cells, Xenopus homologues of the yeast MCM protein family ([5] and references therein). In addition, a number of mammalian homologues of the MCM protein family were also identified, such as the human CDC21, CDC46, CDC47 and P1/MCM3[6],[7], and murine $\mathrm{P} 1$ protein[8].

Interestingly, most of the homologues of the MCM protein family, such as S. pombe CDC21, Xenopus MCM3, CDC21, and human CDC21, CDC46, CDC47 and P1/MCM3, have been found to be located in the nucleus throughout the cell cycle[6-11]. This phenomenon is not consistent with the licensing factor model, in which the licensing factor should disappear from the nucleus after the progression of S phase. Two kinds of explanation for this discrepancy have been proposed. First, regulation of nuclear import of MCM homologues may not be an essential aspect of licensing function[11]. This suggestion is supported by the observation that two populations of MCM homologues exist in the amphibian and mammalian nucleus, exhibiting cell-cycle dependent nuclear/ chromatin binding and phosphorylation. An underphosphorylated form binds tightly to a chromatin structure in late G1 and early S phase. During the progression of S phase, the protein becomes hyperphosphorylated and is displaced from the replicated chromatin, although remaining in the nucleus throughout the cell cycle[6-8],[10]. This explanation, however, can not account for why the permeabilization of G2 nuclear membrane in higher eukaryotic cells is critical to allow replicated nuclei to re-start their DNA synthesis. It might be that other protein factor(s) are needed to help the MCM family to carry out their licensing function, and that this additional factor(s) are excluded by the nuclear membrane[5],[9]. Laskey' s group found that nuclear membrane permeabilization is necessary for Xenopus MCM3 to bind to chromatin although XMCM3 itself can cross the intact nuclear membrane. They proposed a cytosolic "loading factor" to be required for XMCM3 binding activity, and this cytosolic 
"loading factor" would be excluded by nuclear envelope during interphase and can enter the nucleus only during mitosis[9]. Similarly, it was found that a Xenopus protein factor, called RLF-B, contains a DNA-binding activity and is required for licensing function of XMCM3. This factor may not be capable of crossing the nuclear membrane[5]. Recently, several groups have found that CDC6 family is a MCM loading factor[12-14].

It is known that the DNA synthesis in eukaryotic cells initiates in many specific loci of chromosomes. However, the relationship between replication licensing factor and the sites of initiation of replication is unclear. Recent experiments suggested that replication licensing does not take place at specific replication origins. First, with immunofluorescence microscopy it was revealed that human CDC21, CDC46 and P1/MCM3 did not co-localize with sites of DNA replication, they actually bound to unreplicated chromatin, indicating that the licensing factors may only play a role in monitoring unreplicated chromatin rather than participating in replication[7]. In addition, Xenopus CDC21, homologues of MCM4, was not found to co-localize with RPA, a key factor in replication initiation complex assembly[10], implying that XCDC21 is not a component of the complex that assembles on origins of DNA replication. Furthermore, by incubating nuclei isolated from Chinese hamster ovary (CHO) cells at various times during the G1 phase in Xenopus egg extract, Wu and Gilbert found that the early G1phase nuclei started replication randomly, whereas late G1-phase nuclei initiated DNA replication specifically at the same origin of replication utilized by cultured CHO cells. In both cases, however, the efficiency of DNA replication appeared the same, demonstrating that the replication licensing was completed in early G1 phase[15]. These results clearly indicate that replication licensing occurs prior to the determination of the sites where replication will initiate. Perhaps replication licensing converts chromatin into an initiation-competent state, but it is not sufficient to decide where to start DNA synthesis.

\section{Determination of functional origins of DNA replication in eu- karyotes by nuclear/chromatin structures}

The concept of origins of DNA replication is concerned with two different terms, "genetic origin" and "functional origin". The "genetic origin" is defined by cisacting DNA sequences which are capable of directing the autonomous replication of linked DNA sequences. The "functional origin" is the actual site in the chromosomes where DNA replication begins. It is important to keep in mind the difference between these two concepts when we talk about the replication origin of eukaryotic cells.

In the genome of prokaryotic cells, bacteriophages or animal viruses, there is a unique cis-acting sequence as functional origin of replication that interacts with initiation proteins. These origin sequences can also be identified genetically according to their ability to promote the replication of constructed plasmids either by in vivo transformation or by in vitro replication[16]. In most cases, there is a single defined site 


\section{Regulation of eukaryotic DNA replication and nuclear structure}

that serves as both the functional and the genetic origin. In eukaryotic cells, multiple origins are needed for DNA replication, since the genome of eukaryotes is much bigger than that of prokaryotes or viruses[1]. In fact, there are much more genetic origins than that are necessary to replicate the entire eukaryotic genome and not every genetic origin serves as a functional origin in each cell cycle[17]. It has been found that the relationship between the genetic origins and the functional origins in eukaryotic cells is more complicated than that in prokaryotic cells.

Autonomously replication sequences (ARS) in budding yeast have been identified as the genetic origins since they promote high-frequency transformation. However, it has been shown that not all ARS elements that function efficiently in plasmid DNA are utilized in their usual chromosomal contexts. For example, only six of the 14 ARS elements present in a region of chromosome III are chromosomal replication origins [18]. In addition, the function of ARS sequences can be inactivated by artificial translocation to different chromosomal positions[19]. Similar results were also found in fission yeast. Huberman and his colleagues found that if they deleted the stronger origin in ura4 gene region of S. pombe chromosome III, the weaker one near the deleted origin became a stronger origin, which suggests that ARS elements interfere with each other in a hierarchical fashion[20]. Thus, the functionality of ARS elements in yeast chromosomes is controlled by the context where they reside.

Although the identification of genetic origins in simple eukaryotic cells has been successful, the search for genetic origin in higher eukaryotes has lagged far behind. The contradictory view of specific sequences for origins has emerged in the literature on studying metazoan DNA replication[21],[22]. On one side, it was shown that a specific sequence in the human b-globin locus was essential for the initiation of DNA replication, and the deletion of this putative origin sequence resulted in the loss of initiation activity of this region[23],[24]. On the other side, the plasmid transfection assays, which successfully identified prokaryotic ori or yeast ARS, have failed to identify metazoan autonomously replicating sequences. In certain human cell lines this assay has shown that autonomous replication occurs to some degree in all the constructed plasmids, whether using random fragments from the human genome or bacterial DNA sequences[25],[26]. Furthermore, Xenopus eggs or egg extracts can replicate any kind of naked DNA templates and the replication initiates at apparently random sites[27].

Although it is uncertain whether genetic origins of DNA replication exist in higher eukaryotic cells, it has been shown that functional origins of replication do exist in higher eukaryotes. Up to date, there are at least 23 published examples detecting functional origins of replication in metazoan[21]. However, the properties of functional origins in higher eukaryotic cells are still not clear. Various techniques that label nascent DNA strands and relate them to their sites of origin have often defined the function origin to a restricted region (down to $0.5 \mathrm{~Kb}$ ). In contrast, by physical mapping the structure of replication intermediates with 2-dimensional agarose gel electrophoresis 
method, replication bubble structures (initiation sites) were detected throughout broad regions of the genome, suggesting replication starts at a delocalized initiation zone in metazoan chromosomes (up to $55 \mathrm{~Kb}$ ). These two kinds of contradictory results have not been completely reconciled[21].

To open an avenue which could deal with the challenge of understanding the characteristic of metazoan origin of replication, researchers attempted to focus on the role of nuclear/chromatin structure in regulating initiation of eukaryotic DNA replication instead of DNA sequences per se. Recently, the first cell-free system that can initiate metazoan DNA replication preferentially at a physiologically utilized origin has been established[27]. They found that when intact G1-phase CHO nuclei were incubated in Xenopus egg extract, initiation of replication occurred specifically on the same locus as cultured CHO cells, whereas the same egg extract initiated DNA replication randomly when damaged nuclei or naked DNA were provided as substrates, demonstrating that assembly of a specific origin in mammalian chromosomes depends on features of nuclear structure[15],[27]. In addition, Gilbert and his group have revealed an entirely novel origin that is utilized only when a unique nuclear structure is assembled around metaphase chromosomes[28]. Furthermore, although replication initiates at random chromosomal sites in rapid cleavage stage of Xenopus embryos, site-specific initiation of DNA replication occurs at the blastula stage of development[29]. Similarly, the sequence independent replication of plasmids microinjected into Xenopus embryos ceases at the blastula stage[27]. A number of changes in nuclear structure have been observed to occur at this time during Xenopus development. Thus, it was suggested that the change of nuclear structure could restrict the initiation of replication to specific origins in differentiated cells[27]. These findings presented here demonstrate convincingly that the functional origins of eukaryotic DNA replication are dependent upon the particular nuclear structure and chromatin context.

\section{"Replication factories" responsible for carrying out DNA replication}

The underlying meaning of the nuclear structure involved in initiation of replication relates directly to the organization of DNA replication in eukaryotic cells. The classical view of how DNA replication occurs is that replication machinery (multi-protein complex) moves along the immobilized track of DNA templates while it synthesizes DNA. Recently, a body of convincing evidences draw a different picture of the DNA replication process: replication protein complexes, bound to the nuclear matrix (or nucleoskeleton), form immobile "replication factories", through which nascent DNA strands are spooled during the process of the DNA replication[30].

With fluorescent microscopy, researchers showed that when the nuclei were pulse labeled with bromodeoxyuridine or biotinylated deoxyuridine and stained with fluorescent antibodies, S-phase eukaryotic nuclei contain about 100-300 bright-staining DNA replication foci of incorporation, indicating that DNA replication forks are organized into tight clusters[1]. These foci have been visualized with electron microscopy as dense, 
Regulation of eukaryotic DNA replication and nuclear structure

morphologically discrete ovoid macromolecular complexes, termed replication factories [31]. Each replication factory may contain about 40 replication forks and string along nuclear matrix. Moreover, by pulse-chase assay, electron microscopy showed that labeled DNA appears to move away from the replication factories[31]. These results indicate that replication protein complexes are attached to the nuclear matrix and synthesize DNA as the template moves through them. The replication factories appeared at the late G1 phase and quickly became active. They increased in size and decreased in number during S phase progression, coinciding with the change of replication foci of incorporation seen by fluorescent microscopy[32].

The concept of the replication factory is not easily reconciled with the traditional view of the replication machinery. Also, it is difficult to explain how replication proceeds in cell free replication systems (such as SV40 virus in vitro replication system) where no nuclear matrix exists. Although no clear answer has come out yet, some clues are emerging from the experiments. It is known that unwinding of SV40 viral origin of replication is the first step of initiation of DNA replication, which is carried out by SV40 T-antigen. SV40 T-antigen unwinding experiment under in vitro condition showed that two T-antigen hexamers form a double-hexamer complex which unwinds bidirectionally the double strand DNA and results in the extruded loops of unwound single strand DNA[34]. Interestingly, incubation of an antibody against T-antigen before the unwinding reaction can stimulate the formation of double-hexamer, suggesting that the antibody functions as a "nuclear matrix", stabilizing the T-antigen hexamers by crosslinking them[33]. In other words, these results indicate that even simple protein-protein interaction can cause the formation of immobile protein complexes. Recently, it was shown that replication factory may exist even in living prokaryote, Bacillus subtilis, in which eukaryotic membrane structure does not exist. The researchers created a fusion protein containing the catalytic subunit of DNA polymerase and green fluorescent protein, and found that one spot was generally seen in the middle of the cells, indicating that the fusion protein was immobilized while nascent DNA was pulled through[34].

The nuclear matrix was first isolated and characterized from rat liver tissue in 1974, which consists of $10 \%$ nuclear proteins, $1.9 \%$ nuclear DNA, 29\% nuclear RNA and $1.5 \%$ phopholipids[35]. Since then, nuclear matrices have been characterized widely from unicellular organism to higher eukaryotes. The nuclear matrix has been considered a structural framework for nuclear multifunction such as DNA replication, transcription and splicing[30]. Consistent with concept of replication factory, enzymes required for DNA replication have been found associated with isolated nuclear matrix, and replication intermediates have also been found enriched with nuclear matrix ([35] and references therein). In addition, sufficient evidences indicate that DNA is organized into supercoiled loops and attaches to the nuclear matrix when it is engaged in replication[35]. Furthermore, some data suggest that the origins of replication remain attached to the nuclear matrix throughout the cell cycle[35]. 
Wu JR

\section{CONCLUSION}

The works eviewed above indicate that the popular views of regulating initiation of eukaryotic DNA replication should be changed. Among them, one involves the property of replication origins, and the other is concerned with the organization of DNA replication. The prevalent model of the replication origin consists of cis-acting sequences as "replicators" and trans-acting proteins as "initiators" that bind to replicators. However, this model is too simplified compared with the real situation of eukaryotic cells. The specific initiation of DNA replication in eukaryotic cells, especially in metazoan cells, are determined by multiple parameters, which may include not only DNA sequences and proteins but also nuclear/chromatin structure. The aspects of nuclear structure involving in the regulation of initiation of DNA replication are correlated tightly with the phenomenon that DNA replication proteins are immobilized to form replication factories for DNA synthesis. How does the nuclear structure participates in the regulation of DNA replication will become a hot topic in the future.

\section{REFERENCES}

[1] Coverley D, Laskey RA. Regulation of eukaryotic DNA replication. Annu Rev Biochem 1994; 63:74576.

[2] Blow JJ, Laskey RA. A role for the nuclear envelope in controlling DNA replication within the cell cycle. Nature 1988; 332:546-8.

[3] Tye BK. The Mcm2-3-5 proteins: are they replication licensing factors? Trends Cell Biol 1994; 4:1606.

[4] Lei M, Kawasaki Y, Tye BK. Physical interactions among Mcm proteins and effects of Mcm dosage on DNA replication in Saccharomyces cerevisiae. Mol Cell Biol 1996; 16:5081-90.

[5] Chong PJ, Th?mes P, Blow JJ. The role of MCM/P1 proteins in the licensing of DNA replication. TIBS 1996; 21:102-6.

[6] Fujita M, Kiyono T, Hayashi Y, Ishibashi M. hCDC47, a human member of the MCM family. J Biol Chem 1996; 271:4349-54.

[7] Krude T, Musahl C, Laskey RA, Knippers R. Human replication proteins hCdc21, hCdc46 and $\mathrm{P} 1 \mathrm{Mcm} 3$ bind chromatin uniformly before S-phase and are displaced locally during DNA replication. J. Cell Sci 1996; 109:309-18.

[8] Kimura H, Nozaki N, Sugimoto K. DNA polymerase associated proteinP1, a murine homolog of yeast MCM3, changes its intranuclear distribution during the DNA synthetic period. EMBO J 1994; 13:4311-20.

[9] Madine MA, Khoo CY, Mills AD, Musahl C, Laskey RA. The nuclear envelope prevents reinitiation of replication by regulating the binding of MCM3 to chromatin in Xenopus egg extracts. Curr Biol 1995; 5:1270- 9 .

[10]Coue M, Kearsey SE, Mechali M. Chromatin binding, nuclear localization and phosphorylation of Xenopus cdc21 are cell-cycle dependent and associated with the control of initiation of DNA replication. EMBO J 1996; 15:1085-97.

[11]Maiorano D, Assendelft GB, Kearsey SE. Fission yeast cdc21, a member of the MCM protein family, is required for onset of $\mathrm{S}$ phase and is located in the nucleus throughout the cell cycle. EMBO J1996; 15:861-72.

[12]Donovan S, Harwood J, Drury LS, Diffley JF. Cdc6p-dependent loading of Mcm proteins onto prereplicative chromatin in budding yeast. Proc Natl Acas Sci USA 1997; 94:5611-6. 


\section{Regulation of eukaryotic DNA replication and nuclear structure}

[13] Tanaka T, Knapp D, Nasmyth K. Loading of an Mcm protein onto DNA replication origins is regulated by Cdc6p and CDKs. Cell 1997; 90:649-60.

[14] Perkins G, Diffley JF. Nucleotide-dependent prereplicative complex assembly by Cdc6p, a homolog of eukaryotic and prokaryotic clamp-loaders. Mol Cell 1998; 2:23-32.

[15] Wu JR, Gilbert DM. A distinct G1 step required to specify the Chinese hamster DHFR replication origin. Science 1996; 271:1270-2.

[16] De Pamphilis ML. Eukaryotic DNA replication: anatomy of an origin. Annu Rev Biochem 1993; 62: 29-63.

[17] Diffley JF. Once and only once upon a time: specifying and regulating origins of DNA replication in eukaryotic cells. Genes and Dev 1996; 10:2819-30.

[18] Collins I, Newlon CS. Chromosomal DNA replication initiates at the same origins in meiosis and mitosis. Mol Cell Biol 1994; 14:3524-34.

[19] Brewer BJ, Fangman WL. Initiation at closely spaced replicaiton origins in a yeast chromosomes. Science 1993; 262:1728-31.

[20] Dubey DD, Zhu J, Carlson DL, Sharma K, Huberman JA. Three ARS elements contribute to the ura4 replication origin region in the fission yeast, Schizosaccharomyces pombe. EMBO J. 1994; 3:3638-47.

[21] DePamphilis ML. Replication origins in metazoan chromosomes: fact or fiction? BioEssays 1999; 21 : 5-16.

[22] Gilbert DM. Replication origins in yeast versus metazoa: separation of the haves and the have nots. Curr Opin Gen and Dev 1998; 8:194-9.

[23] Aladjem M, Groudine M, BrodyL, Dieken E, Fournier R, Wahl G, Epner E. Participation of the human b-globin locus control region in initiation of DNA replication. Science 1995; 270:815-9.

[24] Aladjem M, Rodewald LW, Kolman J, Wahl G. Genetic dissection of a mammalian replicator in the human b-globin locus. Science 1998; 281:1005-9.

[25] Krysan P, Smith J, Calos M. Autonomous replication in human cells of multimers of specific human and bacterial DNA sequences. Mol. Cell Biol 1993; 13:2688-96.

[26] Masukatam H, Satoh H, Obuse C, Okazaki T. Autonomous replication of human chromosomal DNA fragments in human cells. Mol Biol Cell 1993; 13:1121-32.

[27] Gilbert DM, Miyazawa H, DePamphilis ML. Site-specific initiation of DNA replication in Xenopus egg extract requires nuclear structure. Mol Cell Biol 1995; 15:2942-54.

[28] Lawlis SJ, Keezer SM, Wu JR, Gilbert DM. Chromosome architecture can dictate site-specific initiation of DNA replication in Xenopus egg extracts. J Cell Biol 1996; 135:1-12.

[29] Hyrien O, Maric C, M?hali M. Transition in specification of embryonic metazoan DNA replication origins. Science 1995; 270:994-7.

[30] Cook PR. The organization of replication and transcription. Science 1999; 284:1790-5.

[31] Hoz? P, Hassan AB, Jackson DA, Cook PR. Visualization of replication factories attached to a nucleoskeleton. Cell 1993; 73:361-73.

[32] Hoz? P, Jackson DA, Cook PR. Replication factories and nuclear bodies: the ultrastructural characterization of replication sites during the cell cycle. J Cell Sci 1994; 107:2191-202.

[33] Wessel R, Schweizer J, Stahl H. Simian Virus 40 T-antigen DNA helicase is a hexamer which forms a binary complex during bidirectional unwinding from the viral origin of DNA replication. J Virol 1992; 66:804-15.

[34] Lemon K, Grossman AD. Localization of bacterial DNA polymerase: evidence for a factory model of replication. Science 1998; 282:1516-9.

[35] Berezney R, Mortillaro MJ, Ma H, Wei XG, Samarabandu J. The nuclear matrix: a structural mileu for nuclear genomic function. In: Berezney R, Jeon KW. Eds. Structural and functional organization of the nuclear matrix. Academic Press: New York 1995:1-66. 